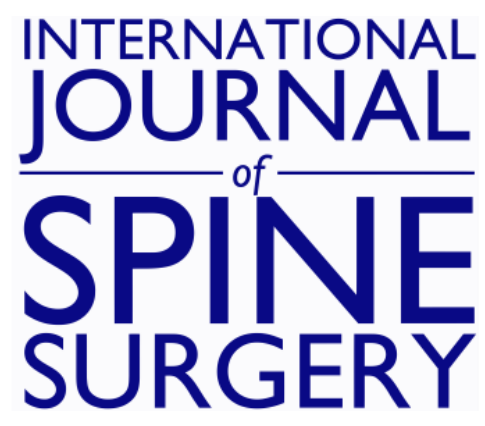

\title{
Recombinant Human Bone Morphogenetic Protein-2 Improves Spine Fusion in a Vitamin D-Deficient Rat Model
}

\author{
VIKAS V. PATEL, ZACHARY R. WUTHRICH, ALICIA ORTEGA, VIRGINIA L. FERGUSON \\ and EMILY M. LINDLEY
}

Int J Spine Surg 2020, 14 (5) 694-705

doi: https://doi.org/10.14444/7101

http://ijssurgery.com/content/14/5/694

This information is current as of April 25, 2023.

Email Alerts Receive free email-alerts when new articles cite this article. Sign up at:

http://ijssurgery.com/alerts 


\title{
Recombinant Human Bone Morphogenetic Protein-2 Improves Spine Fusion in a Vitamin D-Deficient Rat Model
}

\author{
VIKAS V. PATEL, MD, ${ }^{1}$ ZACHARY R. WUTHRICH, BS, ${ }^{1}$ ALICIA ORTEGA, PhD, ${ }^{2}$ VIRGINIA L. \\ FERGUSON, PhD, ${ }^{2}$ EMILY M. LINDLEY, $\mathrm{PhD}^{1}$ \\ ${ }^{1}$ Department of Orthopedics, Divisions of Spine Surgery and Research, University of Colorado Anschutz Medical Campus, Aurora, Colorado, ${ }^{2}$ Department of \\ Mechanical Engineering, University of Colorado Boulder, Boulder, Colorado
}

\begin{abstract}
Background: The effects of vitamin D deficiency on spinal fusion are not well studied, nor are approaches to overcoming deficiency-related detrimental effects. The purpose of this study was to (1) evaluate the effects of vitamin D deficiency on spine fusion in a rat model, and (2) determine whether recombinant human bone morphogenetic protein-2 (rhBMP-2) can improve outcomes in deficient rats.

Methods: Sprague-Dawley rats were assigned to a vitamin D group: vitamin D sufficient (14), vitamin D deficient (16), vitamin D postoperative rescue (15). Posterolateral fusion was performed at L3-4 and L5-6, with one level receiving rhBMP-2 and the other allograft. Following 6 weeks, the spines were harvested for micro-computed tomography (micro-CT) and histological analyses. Fusion was assessed via manual palpation and micro-CT assessment. Micro-CT images were analyzed for bone microarchitecture in intact L5 vertebral bodies and within fused bone masses treated with rhBMP-2.

Results: There were no significant effects of vitamin D status on fusion assessments. However, the microarchitecture of native bone in the intact L5 vertebral bodies of vitamin D-sufficient rats showed significantly greater trabecular thickness $(P<.001)$ and bone volume fraction $(P<.001)$, with decreased trabecular spacing $(P<$ .001), than that of vitamin D-deficient rats. Fusion masses of rhBMP-2 levels also showed significant effects of vitamin D supplementation on both bone volume fraction and trabecular thickness. Histological analysis confirmed that robust bone formation was observed in rhBMP-2-treated fusions, but not in fusion levels treated with allograft.

Conclusions: Overall, vitamin D deficiency decreased trabecular bone microarchitecture, and treatment with rhBMP-2 improved outcomes across all vitamin D groups.

Clinical Relevance: Given the prevalence of vitamin D deficiency in spine surgery patients, vitamin D supplementation may be a cost-effective method for reducing the risk of pseudoarthrosis.

Lumbar Spine

Keywords: vitamin D, lumbar spine, fusion, rat model, rhBMP-2, micro-CT, allograft
\end{abstract}

\section{INTRODUCTION}

Spine fusion procedures are commonly used to treat degenerative spine diseases; however, success rates remain highly variable with pseudoarthrosis rates ranging from $5 \%$ to $34 \% .^{1}$ Multiple factors have been implicated in the failure of spine fusion surgery, including tobacco use, infection, and low bone mineral density. While poor bone quality associated with osteoporosis is known to affect the stability of spine instrumentation and fusion status, little consideration has been given to the role of vitamin D deficiency on spine fusion outcomes.

Vitamin D is a group of fat-soluble prohormones that plays an important role in bone mineralization, overall musculoskeletal health, and fracture prevention. Although there is no widespread consensus on the optimal dosage regimens of vitamin $\mathrm{D}$ for various age groups, vitamin D deficiency is often defined as serum levels of 25-hydroxy vitamin D (25[OH]D) below $20 \mathrm{ng} / \mathrm{mL}$ and vitamin D insufficiency is defined as serum levels between 20 to 30 $\mathrm{ng} / \mathrm{mL}$. Vitamin D deficiency has long been recognized as a common medical problem, particularly in the elderly, that can result from a lack of adequate dietary intake or exposure to ultraviolet B rays, disorders that limit vitamin $\mathrm{D}$ absorption, and conditions that impair the conversion of vitamin D into active metabolites. More recently, however, the prevalence of asymptomatic vitamin D deficiency and insufficiency in otherwise healthy adolescents and adults has received a great deal of attention. Studies have shown that up to $42 \%$ of otherwise 
healthy young adults have low vitamin D levels..$^{2,3}$ Furthermore, 2 recent studies of patients scheduled to undergo spine surgery found that nearly $60 \%$ of patients with spine disorders had insufficient levels of vitamin $\mathrm{D}$ at their preoperative assessment., Thus, vitamin D deficiency is an issue in this patient population that should be considered when evaluating patients for surgery.

The majority of clinical research on vitamin D and skeletal health has focused on the elderly, osteoporotic postmenopausal women, and fracture healing. In general, these studies have shown that serum $25(\mathrm{OH}) \mathrm{D}$ levels are inversely related to the risk for hip and spine fractures ${ }^{6-8}$ and that vitamin D supplementation can improve callus formation and fracture healing. ${ }^{9}$ Several animal studies have also investigated the effects of vitamin $\mathrm{D}$ on fracture healing and found that supplementation with vitamin D increased callus formation, bone remodeling, and trabecular number. ${ }^{10}$ Other animal studies have used a vitamin D-deficient rat model to investigate the effects of low serum vitamin D levels on various aspects of health. Kaastad et al ${ }^{11}$ found that rats maintained on a vitamin $\mathrm{D}$-depleted diet had reduced distal tibial trabecular bone volume and ultimate stress in the femoral shaft. Similarly, Lee et $\mathrm{al}^{12}$ maintained rats on a vitamin D-deficient diet plus various levels of supplemental vitamin $\mathrm{D}_{3}$ and found a significant correlation between circulating levels of $25(\mathrm{OH}) \mathrm{D}$ and femoral trabecular bone volume, L2 vertebral trabecular bone volume, and midshaft tibial cortical bone volume. These studies show that vitamin D plays a role in bone volume and fracture healing, which could suggest that vitamin D deficiency may also increase the risk for pseudoarthrosis after spine fusion surgery.

Thus, the goal of this study was to determine the effects of vitamin D deficiency on posterolateral lumbar fusion in vivo using a rat model, which is an accepted animal model for studying both spine fusion and vitamin D deficiency. ${ }^{12,13}$ Furthermore, given that recombinant human bone morphogenetic protein-2 (rhBMP-2) has been shown to improve outcomes in patients with certain risk factors for pseudoarthrosis, ${ }^{14}$ we also investigated the ability of rhBMP-2 to overcome the potential detrimental effects of vitamin D deficiency on fusion outcomes. We hypothesized that rats maintained on a diet depleted of vitamin D would have poorer fusion outcomes than rats fed a standard diet that includes vitamin D. We further hypothesized that immediate postoperative rescue of vitamin D levels would result in better fusion outcomes than those of rats maintained on the diet depleted of vitamin D. Lastly, we hypothesized that the use of rhBMP-2 would improve fusion outcomes, as compared to allograft alone, in rats with low vitamin D levels.

\section{METHODS AND MATERIALS}

\section{Animals and Dietary Conditions}

All animal procedures were approved by our local Institutional Animal Care and Use Committee. Fifty-seven mature male Sprague-Dawley rats (Harlan Laboratories, Indianapolis, Indiana) weighing approximately $330 \mathrm{~g}$ were randomly assigned to the following groups: vitamin D sufficient (Sufficient; $n=16$ ), vitamin $\mathrm{D}$ deficient (Deficient; $\mathrm{n}=18$ ), vitamin $\mathrm{D}$ rescue (Rescue; $\mathrm{n}=15$ ), vitamin $\mathrm{D}$ sufficient allograft harvest $(\mathrm{n}=3)$, and vitamin $\mathrm{D}$ deficient allograft harvest $(n=5)$. There was no specific randomization procedure, blocks of cages were assigned to a group for ease in diet chow administration.

The Sufficient group was maintained on a diet of standard rat chow (2.0 IU/g vitamin D3; Harlan 2918S) prior to delivery, and throughout the study timeline. The Deficient group was fed a special diet of rat chow devoid of vitamin D (0 IU/g vitamin D3; Harlan TD.89123) at Harlan Laboratories for 8 weeks prior to delivery and then maintained on the diet throughout the study. The Rescue group was maintained on the vitamin $\mathrm{D}$ depleted diet prior to surgery and then transferred immediately postoperative to the standard rat chow. Rats were housed in single cages in a vivarium with a 12-hour light:12hour dark cycle, no ultraviolet light exposure, and food and water ad libitum.

\section{Blood Collection}

Blood was collected under isoflurane anesthesia approximately 1 week prior to surgery via tail vein. A second sample was collected via cardiac puncture immediately after sacrifice. Samples were centrifuged, serum was stored at $-20^{\circ} \mathrm{C}$, and serum levels of $25(\mathrm{OH}) \mathrm{D}$ were later analyzed via radioimmunoassay (DiaSorin Inc., Saluggia, Italy). The lower limit cutoff of the radioimmunoassay was $5.0 \mathrm{ng}$ / $\mathrm{mL}$. Levels below this detectable limit were assigned a value of $4.9 \mathrm{ng} / \mathrm{mL}$ for analyses. 
Table 1. Fusion assessment scale.

\begin{tabular}{|c|c|}
\hline Fusion Score & Corresponding Statements \\
\hline \multicolumn{2}{|l|}{ Not Fused } \\
\hline 0 & No new bone growth \\
\hline 1 & $\begin{array}{l}\text { Little bone formation on both sides, with less than } 25 \% \\
\text { new bone growth between pedicles }\end{array}$ \\
\hline 2 & $\begin{array}{l}\text { Moderate bone formation on one side, with } 26 \%-50 \% \\
\text { new bone growth between pedicles; little bone growth } \\
\text { on the other side with less than } 25 \% \text { new bone growth } \\
\text { between pedicles }\end{array}$ \\
\hline 3 & $\begin{array}{l}\text { Good bone formation on one side, with } 51 \%-75 \% \text { new } \\
\text { bone growth between pedicles; moderate bone } \\
\text { formation on the other side, with } 26 \%-50 \% \text { new bone } \\
\text { growth between pedicles }\end{array}$ \\
\hline \multicolumn{2}{|r|}{ 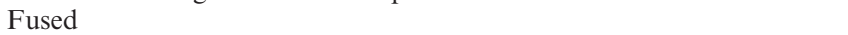 } \\
\hline 4 & $\begin{array}{l}\text { Fusion on one side; good bone formation on the other } \\
\text { side with } 51 \%-75 \% \text { new bone growth }\end{array}$ \\
\hline 5 & Solid union, excellent bone formation on both sides \\
\hline
\end{tabular}

\section{Bone Graft Materials and Harvesting}

Prior to spine fusion surgery, allograft bone from the iliac crest, spine, tibia, and femur was harvested from 8 rats $(\mathrm{n}=5$ Deficient, $\mathrm{n}=3$ Sufficient $)$ immediately after sacrifice. Allograft bone was used to eliminate the need for an additional surgical site and the corresponding comorbidities associated with autograft harvesting. Approximately $0.4 \mathrm{~g}$ of morselized allograft bone was placed in treated levels during posterolateral fusion.

rhBMP-2 (Medtronic Inc, Fridely, Minnesota) was prepared by soaking $5 \times 5 \times 10$-mm collagen sponges with $0.032 \mathrm{mg} / \mathrm{mL}$ rhBMP-2. ${ }^{15}$ All rats received rhBMP-2 at one level and allograft bone at the other (counterbalanced among levels). Separate fascial incisions limited the potential for crosscontamination between levels.

\section{Posterolateral Fusion Surgery}

Rats were anesthetized (4\%-5\% isoflurane) and maintained under continuous anesthesia $(1.5 \%-3 \%$ isoflurane) during L3-L4 and L5-L6 posterolateral intertransverse fusion. Buprenorphine $(0.1-0.5 \mathrm{mg} /$ $\mathrm{kg}$ subcutaneous) was given at anesthesia induction for analgesia. After prepping and sterilizing the lumbar region, a skin incision $(\sim 6 \mathrm{~cm})$ was made on the dorsal lumbar midline. Following exposure of the dorsolumbar fascia, bilateral muscle-splitting incisions were made to expose the transverse processes. A scalpel blade and burr were used to decorticate the transverse processes of L3-4 and L56 and debris was removed via saline irrigation. Extensive precautions were taken to avoid crosscontamination of graft materials between the levels, including separate fascial incisions, which essentially created separate compartments in the spine. After placement of graft material, the facial layer was closed in continuous fashion. Bupivicaine was put into the incision for local pain relief and skin was closed in the standard interrupted fashion. Subcutaneous saline was administered for additional recovery and the rats were kept on a warming blanket while recovering from anesthesia. One rat (Sufficient) died immediately after surgery and 3 others (1 Sufficient, 2 Deficient) died in the perioperative period, leaving final groups: $n=14$ Sufficient, $\mathrm{n}=16$ Deficient, and $\mathrm{n}=15$ Rescue.

\section{Postoperative Procedures}

Buprenorphine (0.1-0.5 mg/kg subcutaneous) was provided at a minimum of every 12 hours for 72 hours postsurgery. After recovery, food and water were provided to all animals ad libitum. For the remainder of the study, chow was placed in the bottom of cages for easier access and to limit unnecessary rearing or extension in the lumbar spine region. Health status was evaluated daily. Sutures were removed at $\sim 3$ weeks postoperatively when the wounds appeared healed. At 6 weeks postoperative, rats were euthanized via overdose of pentobarbital $(150-200 \mathrm{mg} / \mathrm{kg})$. Spines were harvested and prepared for analyses.

\section{Manual Palpation Fusion Analysis}

Gross explanted rat spines underwent standardized manual palpation by 2 independent experienced spine surgeons blinded to the group status. ${ }^{13,15-17}$ Fusion status at the L3-4 and L5-6 segments was determined via bending in the sagittal and coronal planes to test for intersegmental motion. No observable motion at the segment of interest was determined to be fused while any motion was determined to be not fused.

\section{Micro-Computed Tomography Fusion Analysis}

The explanted spines underwent micro-computed tomography (micro-CT) imaging (Siemens Inveon micro-CT, Siemens Medical Solutions, Knoxville, Tennessee; $75-\mu \mathrm{m}$ voxel resolution). Three-dimensional reconstruction with Image $\mathbf{J}$ software ${ }^{18}$ allowed for complete visualization of the spine from any perspective. The reconstructed images were scored on a fusion scale (Table 1$)^{19}$ from 1 to 5 by 2 experienced independent spine surgeons blinded to the group status. Fusion was defined as a score of 4 
or 5; disagreements in fusion status were reviewed for final determination by a third experienced independent spine surgeon.

\section{Micro-CT Bone Microarchitecture Analysis}

Micro-CT images were analyzed using CT Analyzer software (Bruker Corp, Allentown, Pennsylvania) to determine bone morphometry for all explanted spines. Intact L5 vertebral bodies were evaluated for effects of vitamin D deficiency on untreated bone. Trabecular bone morphometry within the L3-4 and L5-6 fusion masses was analyzed to determine the effects of vitamin D deficiency on bony healing following the fusion procedure. Images were thresholded and segmented to produce binary images using custom MATLAB code following recommended guidelines. ${ }^{20}$ Then uniform cylinders containing only trabecular bone, and excluding cortical bone, were carefully selected to encompass volumes within the intact L5 vertebrae of $1.6 \mathrm{~mm}^{2}$ diameter $\times$ maximum possible height within each vertebral body. Separately, regions of interest were evaluated that included fused, but not extant, bone regions within fusions at L3-4 and L5-6. All volumes were evaluated for bone microarchitecture, including bone volume fraction (BV/TV), trabecular thickness (Tb.Th), and trabecular spacing (Tb.Sp) ${ }^{20}$, using National Institutes of Health ImageJ and BoneJ. ${ }^{21}$

\section{Histological Analysis}

Following micro-CT imaging, all spines were dehydrated in a graded series of ethanol, cleared in acetone, and infiltrated with poly(methylmethacrylate) (PMMA). To identify sectioning planes that encompassed each fusion within the PMMA-embedded spines, micro-CT image z-stacks were carefully evaluated to locate the proximal and distal extent of each fusion. The spines were sectioned (Exakt bone saw with a diamond-chip blade; Exakt Technologies, Oklahoma City, Oklahoma), ground to $30-\mu \mathrm{m}$-thick sections using an Exakt precision grinder, and polished to a 1200-grit finish. Sections were stained with Sanderson's Rapid Bone Stain with Van Gieson counterstain (Dorn \& Hart Microede, Loxley, Alabama) and evaluated for maturity of cortical bone surrounding the fusion and trabecular bone within the fusion, as well as for interfaces between the newly formed bone and the bony and soft tissues surrounding and contained within the fusion. Bone maturity was assessed by
Table 2. Serum levels of $25(\mathrm{OH}) \mathrm{D}$ at 1 week preoperative and 6 weeks postoperative. Data are reported as mean $\pm \mathrm{SD}$. $^{\mathrm{a}}$

\begin{tabular}{lccc}
\hline & \multicolumn{3}{c}{ Vitamin D Diet } \\
\cline { 2 - 4 } & Deficient & Rescue & Sufficient \\
\hline $\begin{array}{c}1 \text { week preoperative, } \\
\text { 25(OH)D, ng/ml } \\
6 \text { weeks postoperative, } \\
25(\mathrm{OH}) \mathrm{D}, \mathrm{ng} / \mathrm{ml}\end{array}$ & $4.98 \pm 0.14^{\mathrm{b}}$ & $4.91 \pm 0.03^{\mathrm{b}}$ & $18.60 \pm 2.06$ \\
\hline
\end{tabular}

Abbreviation: 25(OH)D indicates 25-hydroxy vitamin D.

${ }^{\mathrm{a} C}$ Comparisons for treatment (vitamin $\mathrm{D}$ diet) within preoperative and

postoperative time points using 1-way ANOVA with Dunnett post hoc analysis. ${ }^{b} P<.0001$ compared to Sufficient.

evaluating for fibrocartilage, woven bone, and cortical bone.

\section{Statistical Analysis}

Data were analyzed using JMP-7 (SAS Institute Inc. 2009, Cary, North Carolina). $\chi^{2}$ Tests were performed on categorical data, as a function of vitamin D group (Sufficient, Deficient, Rescue) and bone graft material (rhBMP-2, allograft). Nominal logistic regression was used to evaluate manual palpation results for effects of vitamin D diet, graft material, and vertebral level. Analysis of variance (ANOVA) was performed on continuous data, as a function of vitamin D group (Sufficient, Deficient, Rescue) and bone graft material (rhBMP-2, allograft). Post hoc Tukey tests were performed to determine the source of detected significance. Regression analyses were performed to determine the relationship between serum $25(\mathrm{OH}) \mathrm{D}$ levels and fusion status. Cohen's Kcoefficients were determined to measure interrater agreements. All data are reported as mean $\pm \mathrm{SD}$ where $P<.05$ is considered significant.

\section{RESULTS}

\section{Serum 25(OH)D Analysis}

Serum 25(OH)D level analyses confirmed that (1) Deficient and Rescue groups had significantly lower serum vitamin D levels than Sufficient rats $(P<$ .0001) and (2) postoperative Rescue resulted in vitamin D levels at 6 weeks that were comparable to Sufficient rats, and significantly higher than Deficient rats $(P<.0001)$ (Table 2$)$.

\section{Manual Palpation Fusion Analysis}

Manual palpation revealed no significant differences in fusion rates between the vitamin D groups (Table 3). Regardless of vitamin D status, levels 
Table 3. Manual palpation assessment of fusions at 6 weeks postoperative. ${ }^{a}$

\begin{tabular}{lccllc}
\hline & \multicolumn{2}{c}{ Allograft, no./total (\%) } & & \multicolumn{2}{c}{ rhBMP-2, no./total (\%) } \\
\cline { 2 - 3 } \cline { 5 - 6 } Vitamin D Diet & L3-4 & L5-6 & & L3-4 & L5-6 \\
\hline Deficient $(\mathrm{n}=16)$ & $3 / 8(38)$ & $1 / 8(13)$ & & $8 / 8(100)$ & $8 / 8(100)$ \\
Rescue $(\mathrm{n}=15)$ & $5 / 7(57)$ & $0 / 8(0)$ & & $8 / 8(100)$ & $5 / 7(71)$ \\
Sufficient (n=14) & $3 / 7(43)$ & $0 / 7(0)$ & & $7 / 7(100)$ & $4 / 7(57)$ \\
\hline
\end{tabular}

Abbreviation: rhBMP-2 indicates recombinant human bone morphogenetic protein-2.

${ }^{\mathrm{a}}$ Comparisons for treatment (vitamin D diet, graft material, level) using nominal logistic regression showing no effect of diet, a highly significant effect of graft $(P$ $<.0001)$ for both levels, and a significant effect $(P<.01)$ when comparing outcomes across levels.

treated with rhBMP-2 had significantly higher fusion rate than allograft $(P<.0001)$. After combining results across the 2 treated levels and vitamin D groups, 40/45 (89\%) of rhBMP-2 grafted spines were fused at 6 weeks as compared to $12 / 45$ $(27 \%)$ of allograft-treated spines. Independent of vitamin D status, L3-4 levels also had significantly higher fusion rates than those at L5-6 $(P<.01)$. In allograft-treated levels, $11 / 22(50 \%)$ of L3-4 spinal segments fused versus $1 / 23(4 \%)$ of L5-6 segments. In rhBMP-2-treated levels, $23 / 23(100 \%)$ of L3-4 levels fused versus 17/22 (77\%) of L4-6 levels.

\section{Micro-CT Fusion Analysis}

Micro-CT fusion scoring (Table 4) and a panel of representative 3-dimensional reconstructed images (Figure 1) show results that were similar to manual palpation testing. A $\chi^{2}$ test demonstrated no differences in fusion rates among the vitamin D groups. After combining results across the 2 levels and vitamin D groups, rhBMP-2-treated fusion scores were significantly higher than allograft-treated levels (4.58 versus 2.77 , respectively, $P<.0001$ ) and significantly more levels received a score of 4-5, indicating fusion $(88.9 \%$ versus $11.1 \%$, respectively, $P<.0001)$. Mean fusion scores were similar between the 2 levels (3.92 at L3-4 versus 3.42 at L5-6, $P=.053$ ). However, $60 \%$ of fusions at the L3-
4 level received a score of 4-5, indicating a successful fusion, and only 40\% of the L5-6 levels were scored as successfully fused $(P=.009)$.

\section{Micro-CT Morphometry}

\section{L5 Vertebral Bodies}

We verified that grafting material had no influence on bone microarchitecture in the intact L5 vertebral bone and that no graft material was present at this site (Figure 2). Thus data were pooled into the 3 vitamin D groups: Deficient, Sufficient, and Rescue and evaluated for BV/TV, Tb.Th, and Tb.Sp (Figure 3). BV/TV was greatest in rats fed a Sufficient diet and decreased by $47.5 \%(P<$ $.0001)$ in rats maintained on the Deficient diet. $\mathrm{BV} / \mathrm{TV}$ also decreased by $35.1 \%(P<.01)$ in Rescue rats that were transitioned to the standard diet after surgery. Measures of trabecular bone were similarly influenced by diet, with Sufficient rats showing superior outcomes. Specifically, Tb.Th was significantly decreased in both vitamin D Deficient $(-33.8 \%$ decrease, $P<.001)$ and vitamin D Rescue rats $(-33.5 \%$ decrease, $P<.001)$, as compared to the Sufficient group. Similarly, Tb.Sp was significantly increased in vitamin $D$ Deficient rats as compared to Sufficient rats $(+38.5 \%$ increase, $P<$ .0001). Tb.Sp was also increased in Rescue rats, but not significantly more than sufficient rats $(+9.7 \%$, not significant).

\section{Transverse Processes within Fusion Masses}

Qualitative evaluation of reconstructed micro-CT images revealed significant variations in the extent of decortication. While the transverse processes appeared to be consistently decorticated, large portions of the spinous process were also partially or completely decorticated in many of the fusion sites. Quantitative evaluations could not be performed in allograft levels because they lacked

Table 4. Fusion scoring from 3-dimensional reconstructed micro-computed tomography images at 6 weeks postoperative. ${ }^{a}$

\begin{tabular}{|c|c|c|c|c|c|c|c|c|}
\hline \multirow[b]{3}{*}{ Vitamin D Diet } & \multicolumn{4}{|c|}{ Allograft } & \multicolumn{4}{|c|}{ rhBMP-2 } \\
\hline & \multicolumn{2}{|l|}{ L3-4 } & \multicolumn{2}{|l|}{ L5-6 } & \multicolumn{2}{|l|}{ L3-4 } & \multicolumn{2}{|l|}{ L5-6 } \\
\hline & Freq. Fused (\%) & Score & Freq. Fused (\%) & Score & Freq. Fused (\%) & Score & Freq. Fused (\%) & Score \\
\hline Deficient & $1 / 8(13)$ & 2.75 & $0 / 8(0)$ & 2.67 & $7 / 8(88)$ & 4.75 & $8 / 8(100)$ & 4.75 \\
\hline Rescue & $3 / 7(43)$ & 3.50 & $0 / 8(0)$ & 3.00 & $8 / 8(100)$ & 4.88 & $6 / 7(86)$ & 5.00 \\
\hline Sufficient & $1 / 7(14)$ & 2.86 & $0 / 7(0)$ & 2.50 & $7 / 7(100)$ & 4.86 & $4 / 7(57)$ & 4.16 \\
\hline
\end{tabular}

Abbreviations: rhBMP-2 indicates recombinant human bone morphogenetic protein-2; Freq., frequency.

${ }^{\mathrm{a}}$ Data are presented as frequency (ie, number/total), percentage, and mean fusion score. Stepwise analysis showed no effect of fusion level (L3-4 versus L5-6); thus fusion scores were compared for treatments of vitamin D diet and graft material using a $\chi^{2}$ test. No interactions were observed, yet there was a significant effect of graft $(P<$ .001) where fusions with rhBMP showed improvement over those with allograft. 




Figure 1. Three-dimensional micro-computed tomography reconstructions show dorsal views of representative spines from each group in vitamin $D$ Deficient, Rescue, and Sufficient groups with allograft (A; red) and recombinant human bone morphogenetic protein-2 (B; yellow) fusions at both L3-4 and L5-6.

consistent regions of fused bone and failed to demonstrate bridging to the consistency required for region-of-interest declaration (Figures 1, 2, and 4). However, robust bone formation was consistently observed at rhBMP-2-treated fusions, thus regions of interest could be identified and were carefully positioned to include both the cortical shell surrounding the fusion site and trabecular bone within the fusion. Micro-CT assessment of rhBMP2 fusion masses showed that fusions at L3-4 had greater $\mathrm{BV} / \mathrm{TV}(P=.038)$ and improved trabecular microarchitectural measures (Tb.Th, $P<.0001$; Tb.Sp, $P<.05$ ) over fusions at L5-6 (Table 5). Overall, vitamin D status also influenced fusion outcomes, with a 2-way ANOVA revealing effects of vitamin $\mathrm{D}$ treatment on Tb.Th $(P=.019)$ and
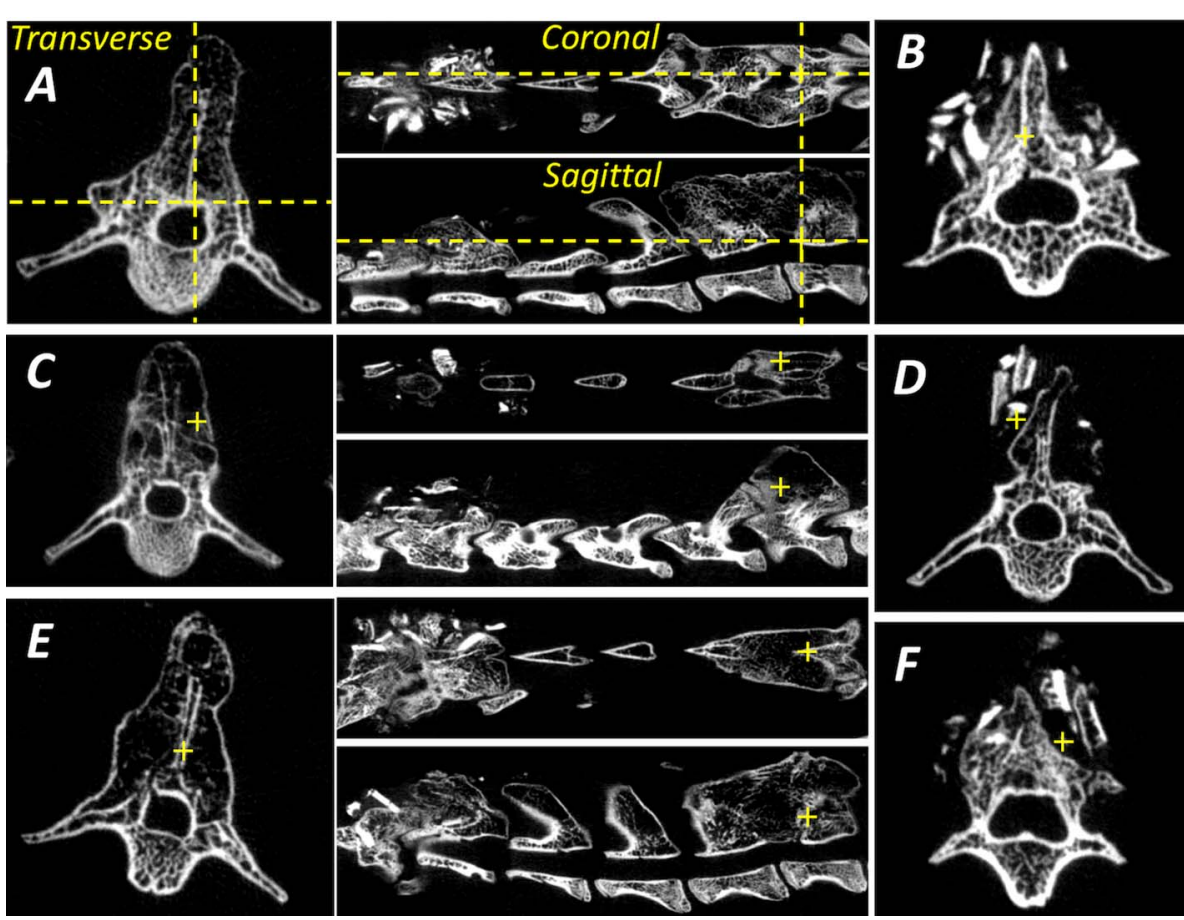

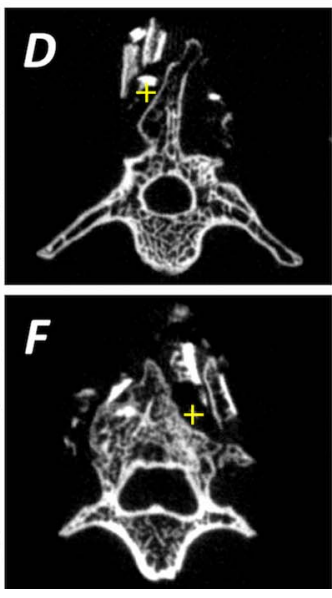

Figure 2. Micro-computed tomography images show representative coronal and sagittal views of spines for rats in vitamin D Sufficient (A, B), Deficient (C, D), and Rescue (E, F) groups. Transverse views spanning each lower lumbar fusion (L5-6, marked with + ) showed robust bone formation with recombinant human bone morphogenetic protein-2 treatment $(A, C, E)$, but not in the presence of allograft $(B, D, F)$. 




Figure 3. Bone microarchitecture outcomes from trabecular bone within intact L5 vertebral bodies to assess influence of dietary vitamin $D$ are plotted as a boxand-whisker plot. Two-way ANOVA $(\alpha=.05)$ demonstrated an effect of diet but no effect of graft (eg, recombinant human bone morphogenetic protein-2 versus allograft), and post-hoc Tukey tests $(\alpha=.05)$ revealed a consistent improvement with vitamin $\mathrm{D}$ sufficiency but not the rescue treatment. All differences are highly significant $(P<.0001)$ and are noted as connecting bars between groups. BV/TV indicates bone volume fraction; Tb.Th, trabecular thickness; Tb.Sp, trabecular spacing.

$\mathrm{BV} / \mathrm{TV}(P=.057)$. At the L3-4 level, vitamin D deficiency significantly decreased Tb.Th by $14.9 \%$ $(P<.10)$ as compared to Sufficient levels, and there was a trend toward decreased BV/TV. Similar trends toward decreased $\mathrm{Tb}$. Th and $\mathrm{BV} / \mathrm{TV}$ were also seen at the L5-6 level of Deficient rats. Overall, Tb.Th, Tb.Sp, and BV/TV in the fusion masses of Rescue rats were similar to Sufficient levels and generally showed improved microarchitecture over Deficient levels.

\section{Histological Analysis}

Histology of fused bone masses confirmed the variable morphology of fusion sites and decortication of the spinous process (Figure 4). Histological analysis also confirmed the observation that robust bone formation was present in rhBMP-2-treated fusions, and discontinuous bone was formed in allograft-treated fusions. In addition, both histological analysis and micro-CT assessments revealed that bone formed within rhBMP-2-treated fusions possessed a thin cortical shell surrounding the fusion and thin trabeculae that contained large gaps filled with marrow. Within allograft fusions, trabeculae were generally thicker than those in the rhBMP-2 fusions and often formed continuous bridges with both the residual spinous process and allograft fragments (Figure 5). However, fused bone in the allograft levels was not consistently bilateral and allograft fragments were often surrounded by soft tissue and not integrated with living bone. Bone masses were present but were often disconnected and possessed irregular morphometry. Both mature (ie, cortical) and immature (ie, porous and wovenlike) bone was present in fused regions of both rhBMP-2- and allograft-treated groups across all vitamin D groups (Figures 4 and 5). Scoring of histological measures produced no discernable pattern in treatment outcomes except to highlight the already noted presence of allograft fragments surrounded by dense connective tissue and the rare presence of small, but inconsistently located, amounts of fibrocartilage.

\section{DISCUSSION}

In this in vivo study, we investigated the effects of vitamin D sufficiency, deficiency, and rescue in a rat L3-4/L5-6 posterolateral spine fusion model, and further compared allograft to rhBMP-2 within each vitamin D group. Overall, treatment with rhBMP-2 led to high rates of fusion, with nearly all treated levels achieving fusion across the vitamin D groups. Three-dimensional reconstructed images showed that fusions using rhBMP-2 had smooth, uniform fusion masses between transverse processes, while fusions using allograft had fusion masses consisting of cortical bone fragments that lacked continuous bone deposition. While the results of fusion assessments did not show a clear influence of vitamin $\mathrm{D}$ status, micro-CT assessment revealed significant effects of vitamin D on trabecular bone microarchitecture. Finally, histological assessment highlighted the visible morphological differences between rhBMP-2- and allograft-treated fusion masses. 


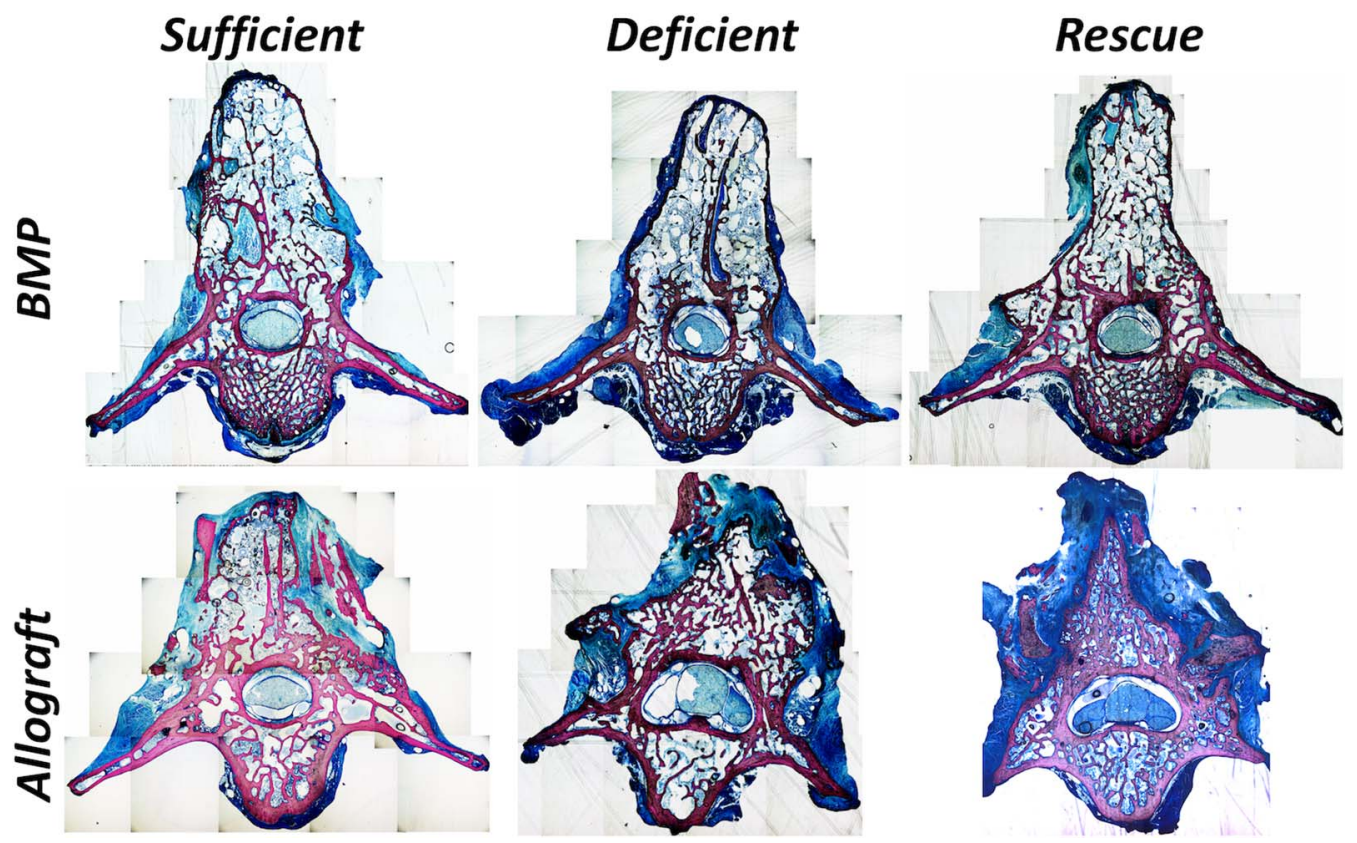

Figure 4. Montaged images ( $\times 5$ magnification) showing regions of fused bone (pink) at L5-6 for Sufficient, Deficient, and Rescue groups are presented. Recombinant human bone morphogenetic protein-2 fusions are denoted by a thin cortical bone-like shell surrounding thin trabeculated bone and marrow (light blue); the partially decorticated spinous process is retained in some samples. Allograft fusions reveal discontinuous bone formation with allograft both forming continuous bone structures with fusion bone as well as allograft fragments surrounded entirely by blue-stained connective tissue.

Although we hypothesized that fusion rates would decrease in rats maintained on the vitamin D-deficient diet prior to and after surgery, the 3 vitamin D diet groups had similar manual palpation and micro-CT fusion scores and rates within each level. The results from manual palpation and microCT fusion assessment suggest that the L5-6 level is a more challenging environment for fusion than that of L3-4: at L5-6 rhBMP fusion success was reduced and none of the allograft-treated rats appeared to be fused on reconstructed micro-CT images. This is likely due to increased stress at the more caudal segment and potential displacement of the graft

Table 5. Microarchitectural outcomes of recombinant human bone morphogenetic protein-2 fusion sites at 6 weeks postoperative. Data are reported as mean $\pm \mathrm{SD}$.

\begin{tabular}{|c|c|c|c|c|}
\hline Level & $\begin{array}{l}\text { Vitamin } \\
\text { D Diet }\end{array}$ & $\mathrm{BV} / \mathrm{TV}, \%$ & Tb.Th, $\mu \mathrm{m}$ & Tb.Sp, $\mu \mathrm{m}$ \\
\hline \multirow[t]{3}{*}{ L3-4 } & Sufficient & $45.1 \pm 6.4$ & $30.2 \pm 3.0$ & $70.6 \pm 16.4$ \\
\hline & Deficient & $39.8 \pm 7.4$ & $25.7 \pm 2.1^{\mathrm{a}}$ & $62.9 \pm 12.6$ \\
\hline & Rescue & $41.7 \pm 6.6$ & $29.2 \pm 2.5$ & $71.1 \pm 6.7$ \\
\hline \multirow[t]{3}{*}{ L5-6 } & Sufficient & $38.8 \pm 10.7$ & $23.7 \pm 3.9$ & $51.3 \pm 15.2$ \\
\hline & Deficient & $29.7 \pm 9.1$ & $22.1 \pm 2.0$ & $64.5 \pm 12.1$ \\
\hline & Rescue & $40.7 \pm 10.0$ & $23.2 \pm 3.5$ & $61.3 \pm 15.2$ \\
\hline \multicolumn{5}{|c|}{ 2-way ANOVA, $P$} \\
\hline Spir & & .038 & $<.0001$ & .030 \\
\hline Vita & & .057 & .019 & N.S. \\
\hline
\end{tabular}

Abbreviations: $\mathrm{BV} / \mathrm{TV}$ indicates bone volume fraction; Tb.Th, trabecular thickness; Tb.Sp, trabecular spacing; N.S., not significant $(P>.10)$.

${ }^{\text {a }} P<.10$ for the Dunnett comparison to the Sufficient control group within each fusion level: L3-4 and L5-6. material away from the transverse process fusion site.

The microarchitecture of intact L5 bodies was assessed on micro-CT reconstructions to evaluate the effects of vitamin D deficiency on untreated bone. After verifying that there was no effect of graft material on extant bone microarchitecture, the data were pooled into the 3 dietary vitamin D groups. This analysis demonstrated a significant effect of dietary vitamin D with Sufficient vertebrae showing higher $\mathrm{BV} / \mathrm{TV}$, increased $\mathrm{Tb}$.Th, and decreased Tb.Sp as compared to Deficient vertebrae. The microarchitecture of intact Rescue vertebral bodies was similar to Deficient vertebrae in terms of $\mathrm{BV} / \mathrm{TV}$ and $\mathrm{Tb}$. Th, and was only superior to Deficient vertebrae in the measure of Tb.Sp. These results indicate that postoperative transition to a standard diet in the Rescue group was insufficient to mitigate most microarchitectural decrements in extant trabecular bone following prolonged vitamin $\mathrm{D}$ deficiency.

Micro-CT reconstructions were also used to score fusion and to evaluate trabecular bone morphometry in the fusion masses between transverse processes. Similar to manual palpation findings, fusion rates were higher in rhBMP-2 versus allograft levels, and were also more successful at the L3-4 level. When evaluating bone microarchitecture of 



Figure 5. Histology images $(\times 5)$ show bone within fusions at L5-6 with recombinant human bone morphogenetic protein-2 and allograft in Deficient, Sufficient, and Rescue groups. Arrows indicate decorticated spinous process, $M=$ marrow, $S=$ skeletal muscle, and $A=$ allograft.

fusion masses, only rhBMP-2-treated levels could be analyzed because allograft-treated levels lacked the bridging bone needed to identify a region of interest. rhBMP-2-treated levels revealed statistically significant effects of vitamin $\mathrm{D}$ diet on $\mathrm{BV} / \mathrm{TV}$ and Tb.Th, with Deficient bone masses showing diminished microarchitectural measures as compared to the Sufficient and Rescue groups. Importantly, these findings show that there were differences in trabecular bone morphometry within the fusion masses even though assessments via micro-CT and manual palpation did not find differences between vitamin D groups. Further, measures of bone microarchitecture in the Rescue group fusion masses were overall similar to those of Sufficient rats. This is in contrast to the findings in intact vertebral bodies, where the quality of trabecular bone in the Rescue group was largely diminished compared to the Sufficient group. This finding indicates that healing bone contained within fusion regions is highly susceptible to circulating vitamin D levels, whereas untreated bone within extant regions was not affected by the short-term increased serum vitamin D levels. This outcome is likely due to little turnover taking place in skeletally mature, intact bone. Postoperative treatment with vitamin D supplementation in this rat fusion model thus effectively reversed detriments in fusion microarchitecture that otherwise would have been seen with continued deficiency.

While histological assessment indicated no clear difference in large bony features with vitamin D status, the morphologic difference between rhBMP$2-$ and allograft-treated tissues was notable. rhBMP-2 graft regions showed robust fusion in decorticated bone with a thin and porous, yet continuous, cortical bone shell surrounding the newly formed bone mass. A sparse network of trabeculae, infilled with marrow, was contained within fused bone masses. Cortical bone was denser on the spinous process, and trabecular bone was thin or absent within marrow regions. Trabeculae commonly extended directly from the spinous process, where present, to form a continuous bony structure. In contrast, fusions with allograft often included a discontinuous cortical shell and thick trabeculae that were observed to integrate with allograft fragments and extant bone (eg, partially decorticated spinous processes); elsewhere allograft fragments were observed to be surrounded only by dense connective tissue. 
The results of serum 25(OH)D analyses indicated that the vitamin D-deficient diet successfully led to very low vitamin D levels, typically less than $5 \mathrm{ng} /$ $\mathrm{mL}$. However, the standard rat chow given to the Sufficient and Rescue groups resulted in vitamin D levels below the range considered sufficient for humans. While serum $25(\mathrm{OH}) \mathrm{D}$ values greater than or equal to $30 \mathrm{ng} / \mathrm{mL}$ are typically considered to be healthy, the rats in our Sufficient and Rescue groups had mean serum levels of 20.6 and $18.6 \mathrm{ng} / \mathrm{mL}$, respectively, at the conclusion of the study. These values are in the "insufficient" range for humans (20-30 ng/mL). Although these serum levels were significantly higher than those of rats maintained on the vitamin $\mathrm{D}$-deficient diet $(4.9 \mathrm{ng} / \mathrm{mL})$, it is possible that greater differences in fusion and microarchitecture outcomes would have been seen if the Sufficient group had achieved vitamin D levels at or above $30 \mathrm{ng} / \mathrm{mL}$. However, it is unclear whether the serum $25(\mathrm{OH}) \mathrm{D}$ reference ranges for humans are also applicable for rodents.

To our knowledge, only 1 other study of vitamin $\mathrm{D}$ effects in a rat posterolateral fusion model has been published, which compared deficient, insufficient, sufficient, and hypervitaminosis D diet groups at 12 weeks following L4-5 posterolateral fusion using tail autograft. ${ }^{22}$ While improvements were observed in fusion and bone morphometry in the hypervitaminosis D group, there were no significant differences between the other groups in manual palpation, radiographic fusion, or micro-CT bone morphometry. In comparison, our fusion model included an earlier postoperative time point (6 weeks) and a Rescue group that was converted to a standard vitamin D diet postoperatively. Whereas the prior study did not find differences in bone morphometry between their sufficient and deficient groups, ${ }^{22}$ we observed diminished bone microarchitecture in Deficient fusion masses. Further, our study showed beneficial effects of the vitamin $\mathrm{D}$ diet on the microarchitecture of untreated bone. Our findings are similar to this prior study in that we also observed no differences in manual palpation or micro-CT fusion rates among groups. Together, these findings suggest that future studies of spinal fusion in rodents should be augmented with bone microarchitectural assessment within the fusion mass.

Limitations of this study include a relatively small sample size and the lack of a high-vitamin D diet. A larger sample size would have reduced the variance and led to improved statistical power for all analyses. The standard chow and vitamin Ddeficient diets had $1 \%$ and $0.47 \%$ calcium and $0.7 \%$ and $0.3 \%$ phosphorus, respectively, and did not control for minerals and nutrients that are important in bone metabolism. We also did not measure circulating levels of calcium or parathyroid hormone. However, our methods are consistent with other studies evaluating dietary modulation of vitamin $\mathrm{D},{ }^{10,11}$ which also did not assess parathyroid hormone and calcium levels. Our micro-CT analyses of fusion mass bone morphometry were limited to rhBMP-2-treated levels because allografttreated levels did not consistently form the bridging bone needed to identify a region of interest. However, given that differences in bone morphometry between vitamin $\mathrm{D}$ groups were seen within the rhBMP-2-treated levels, despite the high fusion rate in this group, it is likely that the control allografttreated fusion masses were similarly influenced by vitamin $\mathrm{D}$ deficiency. There is also a theoretical concern for cross-contamination of the rhBMP-2 and allograft treatments across levels. We could have placed a single treatment in each rat to avoid this concern. However, in an effort to judiciously use animals, we chose instead to create 2 separate fascial incisions to contain the 2 treatments in separate compartments within a single animal. We observed little bone formation at allograft levels, which provides evidence that rhBMP-2 did not affect the allograft-treated levels, and thus there is a low likelihood that cross-contamination occurred in this fusion model. Our results are also limited by the histology methods and staining, which did not allow us to distinguish calcified osteoid from unmineralized osteoid. It is possible that vitamin D-sufficient and -deficient levels both had osteoid present, but that the deficient osteoid was not calcified. Future studies of vitamin D deficiency may consider using histological methods that can detect these differences. Lastly, we chose a 6-week follow-up period to assess the early stages of fusion. It is possible that additional differences between groups would have been detected at later time points.

\section{Conclusion}

This study suggests that systemic vitamin D deficiency leads to diminished bone microarchitecture measures in untreated bone. Moreover, the integrity of bone in rhBMP-2 treated posterolateral fusion masses were adversely affected by extended 
preoperative vitamin D deficiency. Importantly, the detrimental effects of preoperative deficiency on trabecular bone were largely reversed in rats that were converted to a vitamin D diet after posterolateral fusion surgery. Treatment with rhBMP-2 also improved fusion outcomes across all vitamin D groups. Given the prevalence of vitamin D deficiency in spine surgery patients, vitamin D supplementation may be a potential cost-effective approach for improving spine microarchitecture in the perioperative period, during which bone integrity is important for the development of successful fusion. Treatment with rhBMP-2, rather than autograft, may also play a role in overcoming the effects of vitamin D deficiency on fusion. Future basic and human clinical trials are needed to further elucidate the role of vitamin $\mathrm{D}$ and potential modifiers on spine fusion outcomes.

\section{ACKNOWLEDGMENTS}

Portions of this work were funded by Medtronic Inc (Minneapolis, MN), and the Anschutz Foundation (Denver, CO). The authors wish to thank Todd Baldini, MS, Kenneth Milligan, MD, Damian Illing, MD, and Zachary McBeth, MD, for their contributions to the animal work portion of this study. We also wish to thank Zachary Jones, Faye Titchenal, Patrick Muljadi, and Kathryn Thompson from Mechanical Engineering at the University of Colorado and Jian Wei Tay at the University of Colorado BioFrontiers Institute for assistance with micro-CT image segmenting and analysis using custom MATLAB code.

\section{REFERENCES}

1. Muschler GF, Takigami H, Nakamoto C. Principles of bone fusion. In: Herkowitz HN, Garfin SR, Eismont FJ, Bell GR, Balderston RA, eds. Rothman-Simeone: The Spine. Philadelphia, PA: WB Saunders; 2006.

2. Nesby-O'Dell S, Scanlon KS, Cogswell ME, Gillespie C, Hollis BW, Looker AC, Allen C, Doughertly C, Gunter EW, Bowman BA. Hypovitaminosis D prevalence and determinants among African American and white women of reproductive age: third National Health and Nutrition Examination Survey, 1988-1994. Am J Clin Nutr. 2002;76(1):187-192.

3. Tangpricha V, Pearce EN, Chen TC, Holick MF. Vitamin $\mathrm{D}$ insufficiency among free-living healthy young adults. $\mathrm{Am} \mathrm{J}$ Med. 2002;112(8):659-662.

4. Stoker GE, Buchowski JM, Bridwell KH, Lenke LG, Riew KD, Zebala LP. Preoperative vitamin D status of adults undergoing surgical spinal fusion. Spine (Phila Pa 1976). 2013;38(6): 507-515.
5. Lindley EM, Perry J, Patel M, Noshchenko A, Bond A, Burger EL, Cain CM, Patel VV. Vitamin D hypovitaminosis and efficacy of supplementation in spine surgery patients. Curr Orthop Pract. 2019;30(3):250-253.

6. Burgi AA, Gorham ED, Garland CF, Mohr SB, Garland FC, Zeng K, Thompson K, Lappe JM. High serum 25hydroxyvitamin D is associated with low incidence of stress fractures. J Bone Miner Res. 2011;26(10):2371-2377.

7. Cauley JA, Lacroix AZ, Wu L, Horwitz M, Danielson ME, Bauer DC, Lee JS, Jackson RD, Robbins JA, Wu C, Stanczyk FZ, LeBoff MS, Wactawski-Wende J, Sarto G, Ockene J, Cummings SR. Serum 25-hydroxyvitamin D concentrations and risk for hip fractures. Ann Intern Med. 2008;149(4):242-250.

8. Sakuma M, Endo N, Hagino H, Harada A, Matsui Y, Nakano T, Nakamura K. Serum 25-hydroxyvitamin D status in hip and spine-fracture patients in Japan. $J$ Orthop Sci.16(4):418-423.

9. Doetsch AM, Faber J, Lynnerup N, Watjen I, Bliddal H, Danneskiold-Samsoe B. The effect of calcium and vitamin D3 supplementation on the healing of the proximal humerus fracture: a randomized placebo-controlled study. Calcif Tissue Int. 2004;75(3):183-188.

10. Fu L, Tang T, Miao Y, Hao Y, Dai K. Effect of 1,25dihydroxy vitamin D3 on fracture healing and bone remodeling in ovariectomized rat femora. Bone. 2009;44(5):893-898.

11. Kaastad TS, Reikeras O, Halvorsen V, Falch JA, Obrant KJ, Nordsletten L. Vitamin D deficiency and ovariectomy reduced the strength of the femoral neck in rats. Calcif Tissue Int. 2001;69(2):102-108.

12. Lee AM, Anderson PH, Sawyer RK, Moore AJ, Forwood MR, Steck R, Morris HA, O'Loughlin PD. Discordant effects of vitamin $\mathrm{D}$ deficiency in trabecular and cortical bone architecture and strength in growing rodents. J Steroid Biochem Mol Biol. 2010;121(1-2):284-287.

13. Drespe IH, Polzhofer GK, Turner AS, Grauer JN. Animal models for spinal fusion. Spine J. 2005;5(suppl 6):209S$216 \mathrm{~S}$.

14. Glassman SD, Dimar JR 3rd, Burkus K, Hardacker JW, Pryor PW, Boden SD, Carreon LY. The efficacy of rhBMP-2 for posterolateral lumbar fusion in smokers. Spine (Phila $\mathrm{Pa}$ 1976). 2007;32(15):1693-1698.

15. Patel VV, Zhao L, Wong P, Pradhan BB, Bae HW, Kanim L, Delamarter RB. An in vitro and in vivo analysis of fibrin glue use to control bone morphogenetic protein diffusion and bone morphogenetic protein-stimulated bone growth. Spine J. 2006;6(4):397-403; discussion 4.

16. Notani N, Miyazaki M, Toyoda M, Kanezaki S, Ishihara T, Tsumura H. Enhancing the effects of exfoliated carbon nanofibers using bone morphogenetic protein in a rat spinal fusion model. J Orthop Res. 2018;36(11):2892-2900.

17. Patel VV, Zhao L, Wong P, Kanim L, Bae HW, Pradhan BB, Delamarter RB. Controlling bone morphogenetic protein diffusion and bone morphogenetic protein-stimulated bone growth using fibrin glue. Spine (Phila Pa 1976). 2006;31(11):1201-1206.

18. Abramoff MD, Magalhaes PJ, Ram SJ. Image processing with ImageJ. Biophoton Int. 2004;11(7):36-42.

19. Lindley EM, Barton C, Blount T, Burger EL, Cain CM, Seim HB, 3rd, Turner AS, Patel VV. An analysis of spine fusion 
outcomes in sheep pre-clinical models. Eur Spine J. 2017;26(1):228-239.

20. Bouxsein ML, Boyd SK, Christiansen BA, Guldberg RE, Jepsen KJ, Muller R. Guidelines for assessment of bone microstructure in rodents using micro-computed tomography. $J$ Bone Miner Res. 2010;25(7):1468-1486.

21. Doube M, Klosowski MM, Arganda-Carreras I, Cordelieres FP, Dougherty RP, Jackson JS, Schmid B, Hutchinson JR, Shefelbine SJ. BoneJ: free and extensible bone image analysis in ImageJ. Bone. 2010;47(6):1076-1079.

22. Metzger MF, Kanim LE, Zhao L, Robinson ST, Delamarter RB. The relationship between serum vitamin D levels and spinal fusion success: a quantitative analysis. Spine (Phila Pa 1976). 2015;40(8):E458-E468.

Disclosures and COI: The research described in this manuscript was approved by our local Institutional Animal Care and Use Committee.
The institution of one or more of the authors (EML, VVP) has received funding from Medtronic Inc, related to this work.

Corresponding Author: Emily M. Lindley, $\mathrm{PhD}$, Department of Orthopedics, University of Colorado Anschutz Medical Campus, 13001 E. 17th Place, Aurora, CO 80045. Phone: (303) 724-0239; Email: emily.lindley@cuanschutz.edu.

Published 11 November 2020

This manuscript is generously published free of charge by ISASS, the International Society for the Advancement of Spine Surgery. Copyright (c) 2020 ISASS. To see more or order reprints or permissions, see http://ijssurgery.com. 